\title{
A Missed Cause of Malabsorption Syndrome: Total Pancreatic Lipomatosis Detected On Computed Tomography
}

\author{
Gurpreet Singh Sandhu' ${ }^{1}$ Anmol Bhatia ${ }^{2 *}$, Rakesh Kochhar ${ }^{2}$ \\ ${ }^{1}$ Departments of Radiodiagnosis and Imaging, Post Graduate Institute of Medical Education and Research, India. \\ ${ }^{2}$ Departments of Gastroenterology, Post Graduate Institute of Medical Education and Research, India.
}

Received: October 24, 2016; Accepted: December 12, 2016; Published: January 30, 2017

*Corresponding author: Anmol Bhatia, Assistant Professor, GE Radiology Section, Department of Gastroenterology, Post Graduate Institute of Medical Education and Research, Chandigarh, India; Tel: 0091-9914201986; E-mail: anmol_bhatia26@yahoo.co.in

\begin{abstract}
Focal fatty replacement is a common finding in pancreas and may be age related. However, total fatty replacement of pancreas is an uncommon entity, and is known as pancreatic lipomatosis. There is deficiency of exocrine pancreatic enzymes resulting in secondary malabsorption in pancreatic lipomatosis patient. We present a case of pancreatic lipomatosis secondary to chronic pancreatic ductal obstruction by calculi resulting in malabsorption which was detected on computed tomography.
\end{abstract}

Keywords: Pancreatic lipomatosis; Computed tomography; Malabsorption

\section{Introduction}

Pancreatic lipomatosis of pancreas is characterized by adipose tissue replacing the pancreatic acinar cells, although pancreatic duct and islet cells are preserved [1-4]. We present a case of pancreatic lipomatosis secondary to chronic ductal obstruction by calculus in a female being treated for malabsorption likely due to coeliac disease with exact etiology diagnosed after computed tomography (CT).

\section{Case report}

A 43-yrs old woman presented with complaints of loose stools, pain abdomen, decreased appetite, loss of weight from 6 months with no obvious relation of food intake (wheat products etc.) to frequency of stools. Physical examination revealed mild pallor with normal vitals. Routine laboratory investigations revealed anemia (Hemoglobin-9gm/dl) with slightly raise alkaline phosphatase (130 U/L). The anti tissue transglutaminase (Ttg) IgA values were raised (33IU/L) which was repeated in our hospital and were borderline (1.3IU/L). The Antinuclear antibody (ANA) was positive (++ to +++). A duodenal biopsy was done which revealed suspicious for malabsorption and reported as Marsh 3a. Ultrasound of abdomen showed diffusely hyperechoic pancreas with foci of calcification (Figure 1A) with mildly prominent small bowel loops having thickened edematous wall and a possibility of enteritis was kept. Patient was conservatively managed on gluten free diet, however did not show improvement. CT scan of the abdomen revealed the pancreatic tissue replaced by fatty tissue with few intraductal chunky calcific foci in the head region (Figure 1B-1C). A diagnosis of diffuse pancreatic lipomatosis was offered.

\section{Discussion}

Pancreatic lipomatosis is characterized by adipose tissue replacing the pancreatic acinar cells, though there is preservation of pancreatic duct and islet cells [1-4]. There is increase in weight and size and morphologically uniform pancreatic enlargement and almost complete absence of the exocrine tissue of the pancreas [1-4].

It is common in elderly, obese individuals and in some patients with congenital disorders such as cystic fibrosis and Shwachman-Diamond syndrome. Other conditions relate to diffuse fatty replacement of pancreas include Steroid therapy, Diabetes mellitus, Chronic Pancreatitis, Cushing's syndrome, Hemochromatosis and Malnutrition [5,6]. Ultrasonography has limited role and can be misleading due to the overlying gas in the bowel loops causing difficulty in the evaluation of the pancreas and its fatty infiltration, which is associated with increased echogenicity of the pancreas making its differentiation difficult from normally seen retroperitoneal fat [7]. Pancreatic tissue replaced by the fat shows a negative attenuation value on unenhanced CT scan [8]. Chemical shift magnetic resonance

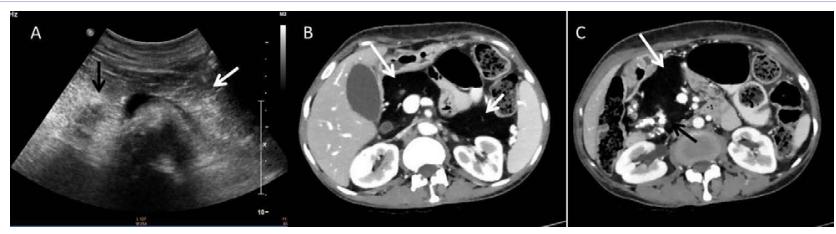

Figure 1: [A-C]: Ultrasound image (A) showing diffusely echogenic pancreas (white arrow) with a discrete echogenic focus in head region (black arrow). CT images (B and C) showing totally fatty replacement of pancreas (white arrows in B and C) with intraductal calcification (black arrow in C). 
imaging (MRI) has its advantage over CT in diagnosing the focal fatty replacement of the pancreatic tissue. There is a characteristic loss of signal seen in opposed-phase T1-weighted gradient-echo image as compared with in-phase image which helps in confirming the presence of microscopic lipid [9].

Marked pancreatic lipomatosis with atrophy has been observed in adult patients secondary to pancreatic duct obstruction by calculi or carcinoma [10]. In these cases, the obstructed pancreatic duct is stenotic rather than dilated, and typically has a smooth wall. Important differentials in case of stenotic pancreatic duct include dorsal agenesis of pancreas, chronic pancreatitis and pancreatic carcinoma [10]. In our case, chronic pancreatic duct obstruction by calculus was the sole etiologic factor responsible for severe degeneration of the pancreatic tissue. We found that the obstructed pancreatic duct was stenotic rather than dilated with complete fatty replacement of the pancreatic tissue.

\section{Summary}

Pancreatic lipomatosis is associated with variable clinical manifestations with malabsorption being one of them Recognition of this entity using CT/MRI is important so that treatment using pancreatic enzyme supplementation along with dietary modification may be initiated.

\section{References}

1. Sharma R, Arora M, Gupta P, Mahajan M, Paul R, Sharma P. A Retrospective Study of Pancreatic Lipomatosis and Its Association with Diabetes Mellitus in General Population in North India. Clinical Medicine and Diagnostics. 2015;5(3):35-38.

2. Patel S, Bellon EM, Haaga J, Park CH. Fat replacement of the exocrine pancreas. AJR Am J Roentgenol. 1980;135(4):843-845.

3. Yasuda M, Niina Y, Uchida M, Fujimori N, Nakamura T, Oono T, et al. A Case of Lipomatous Pseudohypertrophy of the Pancreas Diagnosed by Typical Imaging. JOP. 2010;11(4):385-388.

4. Smits MM, van Geenen EJ. The clinical significance of pancreatic steatosis. Nat Rev Gastroenterol Hepatol. 2011;8(3):169-177.

5. Poddar U, Thapa BR, Sinha SK, Bhasin DK. Massive steatorrhea in a child due to isolated pancreatic hypoplasia. Indian Pediatr. 2001;38(11):1298-1300.

6. Kim KH, Kim CD, Ryu HS, Hyun JH, Chung JP, Chung JB, et al. Endoscopic retrograde pancreatographic findings of pancreatic lipomatosis. J Korean Med Sci. 1999;14(5):578-581.

7. Lee JS, Kim SH, Jun DW, Han JH, Jang EC, Park JY, et al. Clinical implications of fatty pancreas: correlations between fatty pancreas and metabolic syndrome. World J Gastroenterol. 2009;15(15):18691875.

8. Matsumoto S, Mori H, Miyake H, Takaki H, Maeda T, Yamada Y, et al. Uneven fatty replacement of the pancreas: evaluation with CT. Radiology. 1995;194(2):453-458.

9. Kim HJ, Byun JH, Park SH, Shin YM, Kim PN, Ha HK, et al. Focal fatty replacement of the pancreas: Usefulness of chemical shift MRI. AJR Am J Roentgenol. 2007;188(2):429-432.

10. Nakamura M, Katada N, Sakakibara A, Okumura N, Kato E, Takeichi $\mathrm{M}$, et al. Huge lipomatous pseudohypertrophy of the pancreas. Am J Gastroenterol. 1979;72(2):171-174. 